\title{
1 Genetic variability in COVID-19-related genes in the Brazilian
}

3 Rodrigo Secolin ${ }^{1 \dagger}$, Tânia K. de Araujo ${ }^{1 \dagger}$, Marina C. Gonsales ${ }^{1}$, Cristiane S. Rocha ${ }^{1}$, Michel

4 Naslavsky $^{2}$, Luiz De Marco ${ }^{3}$, Maria A.C. Bicalho ${ }^{4}$, Vinicius L. Vazquez ${ }^{5}$, Mayana Zatz ${ }^{2}$,

\section{population}

\author{
Wilson A. Silva ${ }^{6}$, Iscia Lopes-Cendes ${ }^{1 *}$
}

${ }^{1}$ Department of Translational Medicine, University of Campinas (UNICAMP), and the Brazilian

Institute of Neuroscience and Neurotechnology (BRAINN), Campinas, SP, Brazil.

${ }^{2}$ Departament of Genetics and Evolutive Biology, Institute of Bioscience, University of São Paulo,

(USP) and the Human Genome and Stem Cell Research Center, São Paulo, SP, Brazil.

${ }^{3}$ Department of Surgery, Federal University of Minas Gerais (UFMG), Belo Horizonte, MG, Brazil.

${ }^{4}$ Department of Clinical Medicine, Federal University of Minas Gerais (UFMG), Belo Horizonte,

MG, Brazil.

${ }^{5}$ Molecular Oncology Research Center (CPOM), Barretos Cancer Hospital, Barretos, SP, Brazil.

${ }^{6}$ Department of Genetics, Ribeirão Preto Medical School, University of São Paulo at Ribeirão Preto (USP), Ribeirão Preto, SP, Brazil.

$†$ These authors contribute equally to this work

*Correspondence to: Iscia Lopes-Cendes, M.D., Ph.D.

Professor of Medical Genetics and Genomic Medicine

Department of Translational Medicine

School of Medical Sciences

University of Campinas (UNICAMP)

Tessália Vieira de Camargo, 126.

Campinas, SP, Brazil 13083-887

e-mail: icendes@unicamp.br 


\section{ABSTRACT}

3 SARS-CoV-2 employs the angiotensin-converting enzyme 2 (ACE2) receptor and

4 the transmembrane serine protease (TMPRSS2) to infect human lung cells. Previous studies

5 have suggested that different host genetic backgrounds in ACE2 and TMPRSS2 could

6 contribute to differences in the rate of infection or severity of COVID-19. Recent studies

7 also showed that variants in 15 genes related to type I interferon immunity to influenza

8 virus could predispose to life-threatening COVID-19 pneumonia. Additional genes

9 (SLC6A20, LZTFL1, CCR9, FYCO1, CXCR6, XCR1, IL6, CTSL, ABO, and FURIN) and

10 HLA alleles have also been implicated in response to infection with SARS-CoV-2.

11 Currently, Brazil has recorded the third-highest number of COVID-19 patients worldwide.

12 We aim to investigate the genetic variation present in COVID-19-related genes in the

13 Brazilian population. We analysed 27 candidate genes and HLA alleles in 954 admixed

14 Brazilian exomes. We used the information available in two public databases

15 (http://www.bipmed.org and http://abraom.ib.usp.br/), and additional exomes from

16 individuals born in southeast Brazil, the region with the highest number of COVID-19

17 patients in the country. Variant allele frequencies were compared with the 1000 Genomes

18 Project phase 3 (1KGP) and the gnomAD databases. We found 395 non-synonymous

19 variants; of these, 325 were also found in the 1000 Genome Project phase 3 (1KGP) and/or

20 gnomAD. Six of these variants were previously reported as putatively influencing the rate

21 of infection or clinical prognosis for COVID-19. The remaining 70 variants were identified

22 exclusively in the Brazilian sample, with a mean allele frequency of 0.0025 . In silico

23 prediction of the impact in protein function revealed that three of these rare variants were

24 pathogenic. Furthermore, we identified HLA alleles that were previously associated with 
1 COVID-19 response at loci DQB1 and DRB1. Our results showed genetic variability

2 common to other populations, but also rare and ultra-rare variants exclusively found in the

3 Brazilian population. These findings could potentially lead to differences in the rate of

4 infection or response to infection by SARS-CoV-2 and should be further investigated in

5 patients with the disease.

8 Keywords: population genomics, admixed population, COVID-19, SARS-Cov-2 


\section{Introduction}

COVID-19 disease, caused by the SARS-CoV-2 coronavirus, is currently a

3 worldwide pandemic. To enter human lung cells, SARS-CoV-2 employs the spike protein,

4 which is primed by the host serine protease (TMPRSS2), followed by angiotensin-

5 converting enzyme 2 (ACE2) receptor binding, and proteolysis with activation of

6 membrane fusion within endosomes by cathepsin L (CTSL) ${ }^{1-4}$. The main feature in SARS-

7 CoV-2 infection is pre-activation of the spike protein by FURIN inside the host cell, which

8 leads to increased SARS-CoV-2 spread into lung cells and increased virulence ${ }^{5}$. The rapid

9 SARS-CoV-2 infection leads to an exacerbated immune reaction, and a few studies have

10 shown that increased levels of IL-6 (an essential immune response mediator) are associated

11 with increased inflammatory response, respiratory failure, increased probability of

12 intubation, the presence of clinical complications, and higher mortality in patients with

13 COVID-196-8. Additional studies found the enrichment of rare variants predicted to be loss-

14 of-function in genes related to type I interferon (IFN) immunity to influenza virus among

15 patients with life-threatening COVID-19 pneumonia (TLR3, TICAM1, TRIF, UNC93B1,

16 TRAF3, TBK1, IRF3, NEMO, IKBKG, IFNAR1, IFNAR2, STAT1, STAT2, IRF7, and

17 IRF9) ${ }^{9}$.

Specific variants in the $A C E 2$ and TMPRSS2 genes have been reported among

19 diverse populations worldwide, suggesting that different host genetic backgrounds could

20 contribute to differences in COVID-19 infection and severity ${ }^{2,10}$. Ellinghaus et al. ${ }^{11}$

21 performed a genome-wide association study (GWAS) including Italian and Spanish patients

22 with confirmed COVID-19 and controls and identified six candidate genes associated with

23 COVID-19 response on chromosome (chr) 3p21.31 (SLC6A20, LZTFL1, FYCO1, CXCR6, 
$1 X C R 1, C C R 9)$, and one on chr 9q34.2, the locus for genes encoding for the $A B O$ blood

2 group antigens. A subsequent, more extensive study replicated the association between the

3 locus on chr 3p21.31 and COVID-19. It revealed a COVID-19 risk core haplotype ranging

4 from 45,859,651bp to 45,909,024bp, which was inherited from Neanderthals and is

5 currently carried by approximately $50 \%$ of people in South Asia and about $16 \%$ of people

6 in Europe ${ }^{12}$. Interestingly, no evidence of association was found for the previously

7 identified candidate genes that are potentially involved in the response to infection by

8 SARS-CoV-2: ACE2, TMPRSS2, FURIN, and IL6.

$9 \quad$ Furthermore, one significant factor modulating resistance or susceptibility to viral

10 infections is the human leukocyte antigens (HLAs). HLA polymorphism results from a set

11 of amino-acid substitutions in the peptide-binding groove of the HLA molecules that

12 produce variability in the peptide epitope binding-site and presentation to $\mathrm{T}$ cells, which

13 may protect against epidemic infection ${ }^{13}$. Thus, genetic variability in the HLA alleles could

14 influence the immune response in patients with COVID-19, modulating disease severity.

15 Indeed, in silico analysis found that HLA-B*46:01 had the fewest predicted binding sites

16 for SARS-CoV-2 peptides, and HLA-B*15:03 showed the greatest capacity to present

17 highly conserved shared SARS-CoV-2 peptides to immune cells ${ }^{14}$.

18 Brazil has reported the third-highest number of COVID-19 infections worldwide

19 (updated on September 28 ${ }^{\text {th }}$ 2020; https://covid19.who.int/;

20 https://coronavirus.jhu.edu/map.html), and the highest number of cases is concentrated in

21 the south-eastern region of the country (updated on September $28^{\text {th }} 2020$;

22 https://covid.saude.gov.br/). Brazilian individuals feature an admixed genome,

23 encompassing European, sub-Saharan African, and Native Americans as the three main

24 ancestry populations ${ }^{15-17}$, and the distribution of ancestry components varies remarkably 
1 throughout the genome ${ }^{18}$. Furthermore, it has been demonstrated that a significant

2 proportion of genetic variability is still undiscovered in admixed Brazilians ${ }^{19}$ and that

3 genetic variability may lead to differential response to infection ${ }^{20}$. Therefore, we aim to

4 investigate the genetic variation present in COVID-19 related genes in the Brazilian

5 population.

\section{$7 \quad$ Results}

Exome analysis

We found 7,172 variants among the candidate genes analysed (Supplementary Table

10 1). Of these, 395 variants putatively impact protein function, including 354 non-

11 synonymous variants, seven frameshift substitutions, three in-frame deletions, one in-frame

12 insertion, 12 stop gains, two start losses, and 16 splice site variants (Supplementary Table

13 1). Three hundred and twenty-five variants were also present in the gnomAD and/or 1000

14 Genome Project phase 3 (1KGP) databases, including 56 common variants, with an

15 alternative allele frequency $(\mathrm{AAF}) \geq 0.01$ and 269 rare variants $(\mathrm{AAF}<0.01)$

16 (Supplementary Data 1). Although AAF from the admixed Brazilian sample follows the

17 distribution from NFE/EUR, AFR, and AMR in gnomAD and 1KGP databases (Fig. 1), we

18 found differences in the AAF of these common and rare variants in the admixed Brazilian

19 sample compared to gnom $\mathrm{AD}^{21}$ and/or $1 \mathrm{KGP}^{22}$ databases shown in Fig. 1 and

20 Supplementary Data 1. Interestingly, we also observed some variability in the AAF among

21 samples from different Brazilian towns and the two public databases of genomic

22 information on the Brazilian population, BIPMed and ABraOM (Fig. 2). 
More importantly, there were 70 variants which were exclusive to the Brazilian

2 sample, including 11 variants in genes related to type I INF immunity to influenza virus 9 ,

3 six in candidate genes for COVID response identified by GWAS $^{11}$, and five related to

4 SARS-CoV-2 entry in lung cells and virus replication ${ }^{2,10}$. These are rare or ultra-rare

5 variants, presenting a mean AF of 0.0025 (Supplementary Data 1). Among these, we found

6 one in the dataset from Belo Horizonte and two in the ABraOM database for $A C E 2$

7 p.Arg219Cys; one in the dataset from Barretos and two in the ABraOM database for $A C E 2$

8 p.Leu731Phe; and the TMPRSS2 p.Val160Met variant was present in samples from all the

9 different Brazilian towns and the two public databases (BIPMed and ABraOM), with an

10 AAF ranging from 0.1333 in Belo Horizonte to 0.2931 in Campinas. Among the reported

11 variants in genes influencing type I INF immunity to influenza virus ${ }^{9}$, we found three

12 variants in the ABraOM database (one TLR3 p.Pro554Ser, one IFR3 p.Asn146Lys and one

13 IRF7 p.Pro246Ser) (Supplementary Data1 and 2).

14 In addition, we identified five variants (rs35044562, rs34326463, rs35508621,

15 rs67959919, and rs35624553) which were previously described in the COVID-19 risk core

16 haplotype and inherited from Neanderthals ${ }^{12}$. These were only present in samples from

17 Ribeirão Preto and the BIPMed dataset (rs34326463), Campinas (rs35044562, and

18 rs35508621), and the ABraOM dataset (rs35044562, rs35508621, rs67959919, and

19 rs35624553) (Table 1).

21 In silico predictions

We identified seven variants that were predicted to affect protein function for the 12

23 algorithms used: p.Phe249Ser, p.Gly164Val, and p.Leu25Pro in the SLC6A20 gene; 
1 p.Leu96Arg in LZTFL1; p.Tyr287Ser in XCR1; and p.Gly146Ser and p.Asn414Ser in the

2 FURIN gene (Table 2). Furthermore, the variant p.Gly146Ser in the FURIN gene was

3 predicted to destabilise the protein $(\Delta \Delta \mathrm{G}:-1.576 \mathrm{kcal} / \mathrm{mol})$. We observed that the

4 p.Phe249Ser variant is present in samples from Barretos, the BIPMed dataset, gnomAD,

5 and 1KGP (NFE/EUR, AFR, AMR, and SAS populations), whereas the p.Gly164Val

6 variant is present in the ABraOM dataset, gnomAD, and 1KGP (NFE/EUR populations),

7 and the p.Gly146Ser variant is present in the ABraOM dataset, gnomAD, and 1KGP

8 (NFE/EUR, AFR, AMR, EAS, and SAS populations). Notably, four of the variants

9 predicted to be deleterious are found exclusively in admixed Brazilian individuals

10 (p.Leu25Pro in Barretos; p.Leu96Arg in the ABraOM dataset; p.Tyr287Ser in Belo

11 Horizonte; and p.Asn414Ser in the BIPMed dataset).

12 We did not find any predicted deleterious variants in ACE2 and TMPRSS2 based on

13 our 12 algorithm criteria. However, Hou et al. ${ }^{2}$ applied only Polyphen 2 and CADD scores

14 to variants in ACE2 and TMPRSS2 (Polyphen2 $>0.96$ and CADD $>20$ as the cut-off).

15 Therefore, only variants defined as 'probably damaging' by Polyphen2

16 (http://genetics.bwh.harvard.edu/pph2/dokuwiki/overview) and CADD (>20) were

17 included. We found 79 variants predicted to affect protein function, including the

18 p.Val160Met variant in TMPRSS2 reported by Hou et al. ${ }^{2}$, and the p.Pro554Ser variant in

19 TLR3 previously reported by Zhang et al. ${ }^{9}$ (Supplementary Data 2 ).

21 HLA analysis

22 Overall, we identified 331 different HLA alleles in the admixed Brazilian samples.

23 Of these, three HLA alleles have been previously associated with COVID-19 response ${ }^{14,23}$. 
1 We compared the frequency of these HLA alleles in admixed Brazilians and in populations

2 that occupy the top 10 positions with most cases of COVID-19 and the five populations less

3 affected by the disease, including the United States, India, Russia, Colombia, Peru, Mexico,

4 Spain, Argentina, South Africa, Japan, Australia, South Korea, Vietnam, and Taiwan. The

5 frequency of these alleles is described in Supplementary Data 3. We noticed that the HLA-

6 B*46:01, HLA-B*27:07, HLA-B*15:27, and HLA-C*07:29 alleles were absent in the

7 Brazilian samples. The HLA-C*07:29 allele was also absent from other populations and is

8 present at a low frequency $(\mathrm{AF}=0.0003)$ in the Indian population. HLA-B*15:27 was

9 identified in Vietnam, Taiwan, Japan, with an AF $>0.01$ and Spain with AF $<0.0001$. HLA-

10 B*27:07 was detected at a low frequency in India, Colombia, Spain, and South Africa. The

11 HLA-B*46:01 allele was detected in Russia, Mexico, Vietnam, Taiwan, and Japan.

12 Sixty-six Brazilian individuals (17.1\%) presented the HLA-DQB1*06:02 allele

$13(\mathrm{AF}=0.08938), 47$ individuals $(12.2 \%)$ carry the HLA-DRB1*15:01 allele $(\mathrm{AF}=0.06477)$

14 and 32 individuals (8.29\%) have both the HLA-DRB1*15:01 and HLA-DQB1*06:02

15 alleles. The population of other continents, except Oceania, also have these two HLA

16 alleles (HLA-DRB1*15:01 and HLA-DQB1*06:02) with an AF >0.01. Also, 15 Brazilian

17 individuals $(3.88 \%)$ carry HLA-B*15:03 $(\mathrm{AF}=0.02073)$, which is predicted to have the

18 greatest capacity to present SARS-CoV-2 peptides to immune cells ${ }^{14}$. This allele was not

19 found in the Asian population of Japan, South Korea, and Vietnam (Supplementary Data 3).

21 In silico analysis of viral peptide-HLA class I and II binding affinity

22 To verify the potential for an HLA allele type to produce an antiviral response to

23 SARS-CoV-2, we performed HLA binding affinity analyses to the SARS-CoV-2 proteome. 
1 We tested 42 HLA-A proteins, 77 HLA-B, 38 HLA-C, 60 HLA-DP (DPA1/DPB1), 145

2 HLA-DQ (DQA1/DQB1), 46 HLA-DRB1, 4 DRB3, 2 DRB4, and 6 DRB5.

The SARS-CoV-2 proteome was presented by a diversity of HLA alleles from

4 classes I and II (Supplementary Table 2). The HLA proteins are predicted to bind a small

5 proportion of all possible SARS-CoV-2 derived peptides with high affinity (on average

$6 \quad 0.5 \%$ for HLA class I and 2\% for HLA class II). Also, we found a small proportion of weak

7 binders (on average, $1.5 \%$ for HLA class I and 8.2\% for class II). Most of the HLA proteins

8 do not bind either Class I (on average $>96 \%$ ) or class II (on average $>89 \%$ ) molecules

9 (Supplementary data 4). Supplementary Data 5 shows a list of HLA strongest binders

10 (>300 peptides bound at high affinity) of SARS-CoV-2 peptides. These are found in loci

11 HLA-A, -B, -C, and DQ.

\section{Discussion}

Accessing the genomic sequences of the general population is relevant to identify

15 the genetic variability involved in the molecular mechanisms of infection ${ }^{20}$. Also, it is

16 known that the admixed Brazilian population is underrepresented in large public

17 databases ${ }^{21,22}$, and previous studies revealed variants present exclusively in Brazilian

18 indivuduals ${ }^{19,24}$.

19 We studied 27 human COVID-19-related genes and the HLA region in two public

20 genomic databases of admixed Brazilians (BIPMed, www.bipmed.org ${ }^{19}$; ABraOM

21 http://abraom.ib.usp.br ${ }^{24}$ ), and additional samples from individuals born in three different

22 towns of south-eastern Brazil. We reported the variants and HLA alleles found in these

23 samples and compared them with worldwide populations. We also reported variants 
1 constituting the COVID-19 risk core haplotype on locus $3 \mathrm{p} 21.31$, described as being

2 inherited from Neanderthals ${ }^{12}$.

Previous studies showed that the ACE2, TMPRSS2, CTSL, FURIN, and IL6 genes,

4 as well as the HLA region, may be involved in SARS-CoV-2 infection ${ }^{1-5,10}$ and immune

5 response $\mathrm{e}^{6-8,14,23,25}$. Furthermore, variants on loci 3p21.31 and 9q34.2 (encompassing

6 SLC6A20, LZTFL1, FYCO1, CXCR6, XCR1, CCR9, and ABO) have been associated with

7 Spanish and Italian patients with COVID-19 11 , and different variants were found to affect

8 the predisposition to life-threatening illness in patients with COVID-19 from different

9 ancestries ${ }^{9}$.

10 The analysis of genetic variability in candidate genes for specific populations can

11 help to identify individuals at a higher risk of infection or severe disease by constructing

12 risk haplotypes, which can also provide therapeutic targets for the development of more

13 effective treatments and the control of COVID-19 $9^{2,10}$. Thus, in addition to investigating

14 genetic variability in the 27 candidate genes, we extended our analysis to include HLA

15 alleles, which influence immunological response to many infectious agents (updated on

16 September $28^{\text {th }}$ 2020; https://covid19.who.int/; https://coronavirus.jhu.edu/map.html). This

17 is the first comprehensive study of genetic variability of COVID-19 genes in admixed

18 individuals from Latin America, a hard-stricken population in the COVID-19 pandemic ${ }^{26}$,

19 both in terms of the number of infected individuals and the severity of disease leading to

20 increased death rates. Indeed, in the USA, remarkable disparities of SARS-CoV-2 infection

21 by ethnicity have been shown, with Hispanic/Latino and African American individuals

22 presenting higher SARS-CoV-2 infection rates and risk mortality than 'non-Hispanic

23 white' Americans ${ }^{27-29}$. Therefore, by looking at population genomics data, one may gain

24 insights into disease-related variants, which could be disproportionally represented in 
1 specific populations ${ }^{18,30-32}$. Furthermore, by evaluating individuals with unknown

2 information on SARS-CoV-2 infection, one can achieve the random distribution of these

3 variants, allowing better estimates of the distribution of population allele frequencies.

$4 \quad$ We identified small differences in AF in the 395 candidate variants identified

5 among Brazilian samples, strengthening the hypothesis that different genetic backgrounds

6 could influence SARS-CoV-2 infection and behaviour in human host cells ${ }^{2,10}$. Furthermore,

7 this study and previous works ${ }^{2,10}$ identified individuals who carry unique deleterious

8 variants, which may influence gene function and could potentially lead to different

9 responses to SARS-CoV-2 infection on an individual scale. However, the rather similar

10 distribution of AFs among Brazilians and their ancestry populations (NFE/EUR and AFR),

11 as well as other admixed Americans (AMR), and the fact that the unique variants identified

12 in the Brazilian population are rare or ultra-rare, indicates that the admixed Brazilian

13 genetic background is not sufficient to influence SARS-CoV-2 infection on a population

14 scale.

Zeberg and Pääbo ${ }^{12}$ have shown that the major genetic risk factor for severe

16 COVID-19 was inherited from Neanderthals ${ }^{12}$. This finding is important on a regional

17 scale, since 4\% of admixed Americans analysed by Zeberg and Pääbo ${ }^{12}$ (including 1533

18 Brazilian controls from the BRACOVID dataset) presented the core haplotype derived from

19 Neanderthals. Interestingly, Campinas, Ribeirão Preto, and the BIPMed dataset showed

20 only one risk allele, while Barretos and Belo Horizonte did not present any risk allele of the

21 Neanderthal's core haplotype reported. Therefore, if further studies demonstrate that the

22 Neanderthal-derived region confers a risk to COVID-19, this information should be

23 carefully evaluated in additional admixed Brazilian samples from different geographic

24 areas. 
Currently, there is no consensus regarding a possible association of HLA alleles and

2 susceptibility to COVID-19. Ellinghaus et al. ${ }^{11}$ did not find any evidence of an association

3 between HLA and COVID-19. On the other hand, HLA-DRB1*15:01, HLA-DQB1*06:02,

4 and HLA-B*27:07 alleles were associated with Italian patients affected by an extremely

5 severe or severe form of COVID-19 ${ }^{23}$, and an increased frequency of HLA-C*07:29 and

6 HLA-B*15:27 was detected in Chinese patients with COVID-19 in comparison to the

7 Chinese control population ${ }^{25}$. Interestingly, the HLA-C*07:29 allele is absent from the

8 Brazilian admixed samples included in the present study and in all populations used in the

9 comparisons, except for individuals from India, where this allele was found at a low

10 frequency $(0.0003)$. On the other hand, the HLA-B*15:27 allele was identified in

11 individuals from three Asian countries (Vietnam, Taiwan and Japan) with AF $>0.01$, and at

12 a low frequency in Spain (0.0001), but absent from Brazilian samples. The HLA-B*27:07

13 allele found in Italian individuals with a severe manifestation of COVID-19 was also

14 identified in India, Colombia, Spain, and South Africa, but not in populations from Asia

15 and Oceania (countries that are less affected by COVID-19) and from Brazil. In contrast,

16 the HLA-DQB1*06:02 is present in all populations surveyed in this study, including

17 Brazilian individuals (17.1\%), with the exception of individuals from Australia. Also, the

18 HLA-DRB1*15:01 allele is present in all populations investigated in this study, including

19 Brazilian individuals (12.2\%), but not in individuals from Australia and Peru. Interestingly,

$208.29 \%$ of Brazilian individuals carry both the HLA-DRB1*15:01 and HLA-DQB1*06:02

21 alleles.

22 Furthermore, the HLA-A, -B, -C, and DQ loci show haplotypes that are strong

23 binders of SARS-CoV-2 peptides in the Brazilian samples, especially for the HLA-A locus

24 (20 alleles, Table 3). When comparing different populations, we found marked variability 
1 in the frequency of the different HLA alleles putatively associated with the severe

2 manifestation of COVID-19, such as HLA-DRB1*15:01, HLA-DQB1*06:02, and HLA-

$3 \mathrm{~B}^{*} 27: 07$ alleles $^{23}$. Overall, $10 \%$ of Brazilian individuals carried at least two of the alleles

4 associated with the severe manifestation of COVID-19. Interestingly, the same alleles were

5 absent from individuals from Australia. Variability in the frequency of HLA alleles

6 previously associated with COVID-19 highlights the importance of considering ethnic and

7 geographic origin when performing studies investigating the role of HLA alleles and

8 disease. Thus, it seems likely that different population-specific haplotypes may be

9 associated with an increased risk of severe disease in different populations.

10 In conclusion, we found rare variants in three COVID-19-related genes that are

11 present only in the Brazilian dataset and are predicted to affect protein function.

12 Furthermore, we identified HLA alleles previously associated with COVID-19

13 immunological response and 31 HLA alleles predicted as strong binders to SARS-CoV-2

14 peptides at loci -A, -B, -C, and DQ, which indicates the importance of further investigation

15 on the role of HLA haplotypes as modulators of response to infection to SARS-CoV-2.

16 Although the variants predicted to affect protein function in COVID-19-related genes are

17 rare in admixed Brazilians (varying from 0.0001 to 0.0032 ), these also emerge as

18 candidates for modulating response to infection by the SARS-CoV-2 in the Brazilian

19 population. Furthermore, our study suggests the utility of population genomic studies in the

20 context of precision health to stratify risk for infection disorders. 


\section{Methods}

\section{Subjects}

3 We evaluated exomes from 257 individuals from the BIPMed dataset ${ }^{19}, 609$ from

4 the $\mathrm{ABraOM}$ dataset $^{24}$, and an additional 88 exomes from individuals born in three towns in

5 south-eastern Brazil: Barretos $(\mathrm{N}=30)$, Ribeirão Preto $(\mathrm{N}=30)$, located in the state of São

6 Paulo, and Belo Horizonte $(\mathrm{N}=28)$ the capital of the state of Minas Gerais. Among the

7 BIPMed individuals, 193 had information about their city of birth available. The HLA

8 region was sequenced in 386 individuals, including the 257 from BIPMed, the 88 additional

9 exomes, and an additional 41 individuals (22 from southeast Brazil). We signed terms of

10 data privacy to obtain permission to use the raw data from BIPMed and ABraOM public

11 databases and use raw data of the 88 exomes from Barretos, Ribeirão Preto, and Belo

12 Horizonte. This study was approved by the University of Campinas Research Ethics

13 Committee (UNICAMP, Campinas, São Paulo, Brazil). All methods were performed

14 according to the relevant guidelines and regulations.

Whole exome data were stored in variant call format (VCF) files built-in

18 GRCh37/hg19 assembly. Gene regions were extracted by $v$ fftools ${ }^{33}$ based on the position

19 reported in Ensembl GRCh37 Release $101^{34}$ (Supplementary Table 1). Variant

20 consequences were annotated from each gene region by ANNOVAR software (version

212019 Oct 24$)^{35}$, using the following flags: -otherinfo (to include Brazil AF); -onetranscript; -

22 buildver hg19; -remove; -protocol refGene,gnomad211_exome; ALL.sites.2015_08;

23 EUR.sites.2015_08; AFR.sites.2015_08; AMR.sites.2015_08; EAS.sites.2015_08; 
1 SAS.sites.2015_08; -operation g,f; and -nastring. ANNOVAR software provides allele

2 frequency (AF) information from African/African-American (AFR/AFA), Latino/admixed

3 American (LAT/AMR), East Asian (EAS), non-Finish European (NFE), and South Asian

4 (SAS) populations from gnomAD exome dataset ${ }^{21}$, as well as sub-Saharan Africans (AFR),

5 Europeans (EUR), admixed Americans (AMR), east Asians (EAS), and south Asians (SAS)

6 from 1 KGP phase 3 dataset $^{22}$. In addition, we annotated variants which were not identified

7 by ANNOVAR using Variant Effect Prediction (VEP) algorithm ${ }^{36}$, with the following

8 parameters: --buffer_size 500; --canonical; --distance 5000; --species homo_sapiens; --

9 symbol.

To evaluate whether regional variability is observed among Brazilian samples, we

11 separated the samples based on the city in which individuals were born, including 32

12 individuals from Campinas extracted from the BIPMed dataset.

14 In silico prediction analysis

15 To predict the impact on protein function of the non-synonymous variants

16 identified, we applied the following computer algorithms, which is currently recommended

17 by the ACMG/AMP guidelines: PANTHER ${ }^{37}$, MutationTaster $^{38}$, Condel $^{39}$, PROVEAN ${ }^{40}$,

18 PolyPhen2 ${ }^{41}$, Sort Intolerant from tolerant (SIFT) ${ }^{42}$, Align Grantham Variation/ Grantham

19 Difference score (GVGD) ${ }^{43}$, Combined Annotation Dependent Depletion (CADD) ${ }^{44}, \mathrm{PhD}-$

$20 \mathrm{SNPg}^{45}$, Functional Analysis through Hidden Markov Models (FATHMM) ${ }^{46}, \mathrm{SNPs}_{\mathrm{SGO}}{ }^{47}$,

21 and MutPred2 (http://mutpred.mutdb.org).

22 For Align-GVGD, we classified the variants based on the graded classifier with a

23 cut-off of C35 or higher for deleterious classification. For CADD, we used the PHRED-like

24 score with a cut-off of 20 , below which the variants were classified as benign and otherwise 
1 deleterious. For MutPred2, we considered a score threshold of 0.50 for pathogenicity. For

2 all other algorithms, we considered the classification provided as an output.

To access the impact of mutations on protein dynamics and stability, we used the

4 DynaMut server (http://biosig.unimelb.edu.au/dynamut/) ${ }^{48}$. The server requires an input file of protein structure in PDB format or by providing the four-letter accession code for any

6 entry on the Protein Data Bank database (PDB; http://wwpdb.org). The code for the FURIN

7 gene used was $5 \mathrm{jxg}$. The other proteins are not available in the PDB database to be tested.

$9 \quad$ HLA analysis

We sequenced 11 HLA Loci (HLA-A, HLA-B, HLA-C, HLA-DRB1, HLA-DQB1,

11 HLA-DPB1, HLA-DQA1, HLA -DPA1, HLA-DRB3, HLA-DRB4, HLA-DRB5) in 298

12 samples using NGSgo ${ }^{\circledR}$ panels (GenDx, Utrecht, The Netherlands). The DNA libraries

13 were loaded onto a MiSeq Sequencer (Illumina Inc., San Diego, CA, USA), and the data

14 were analysed with the NGSengine v.2.16.2 software (GenDx, Utrecht, The Netherlands).

15 We determined the HLA alleles from the remaining 88 exomes using the HLA-HD (HLA

16 typing from High-quality Dictionary) tool v.1.3.0 ${ }^{49-51}$. The IPD-IMGT/HLA database

17 release 3.40.0 ${ }^{52}$ was used as a reference. Even though we obtained results with six- and

18 eight-digit precision, we restricted the results to four-digit accuracy to compare with

19 published data. HLA allele frequencies were calculated by Arlequin v.3.5.2.2 software ${ }^{53}$.

21 In silico analysis of viral peptide-HLA class I and II binding affinity 
1 corresponding proteins HLA-A, HLA-B, HLA-C, respectively. The HLA-DR is

2 represented by HLA-DRA/DRB1 dimer. Since HLA-DRA is considered monomorphic, we

3 just used the HLA-DRB1. The HLA-DP and DQ are represented by the HLA-DPA1/DPB1

4 dimer and HLA-DQA1/DQB1 dimer, respectively.

FASTA-formatted protein sequence data was retrieved from the National Center of

6 Biotechnology Information (NCBI) database

7 (https://www.ncbi.nlm.nih.gov/genome/browse\#!/overview/Sars-cov-2). The follow eleven

8 protein viral product was used in the analyses: ORF1ab (YP_009724389.1), Surface

9 Glycoprotein (S) (YP_009724390.1), ORF3a (YP_009724391.1), Envelope (E)

10 (YP_009724392.1), Membrane Glycoprotein (M) (YP_009724393.1), ORF6

11 (YP_009724394.1), ORF7a (YP_009724395.1), ORF7b (YP_009725318.1), ORF8

12 (YP_009724396.1), Nucleocapsid (N) (YP_009724397.2), and ORF10 (YP_009725255.1).

13 We k-merised these sequences into 8- to 12-mers to assess HLA class I-peptide

14 binding affinity and into 15-mers to assess HLA class II binding affinity across the entire

15 proteome. Predictions for HLA were performed using different HLA alleles found in our

16 population with netMHCpan v4.1 for class I ${ }^{54}$ and NetMHCIIpan - 3.2 for class II ${ }^{55}$.

HLA allele and haplotype frequencies of other populations

19 HLA frequency data were obtained from the Allele Frequency Net Database

20 (http://www.allelefrequencies.net/) ${ }^{56}$ for 10 distinct populations that are most and least

21 affected by COVID-19. We checked the HLA of the populations that occupy the top 10

22 positions (USA, India, Brazil, Russia, Colombia, Peru, Spain, Mexico, Argentina, South

23 Africa) and those that were less affected (Australia, Vietnam, Taiwan, Japan, and South 
1 Korea) (accessed on 04/24/2020, https://www.worldometers.info/coronavirus/) according to

2 the availability of this data in the Allele Frequency Net Database.

4 Data availability

5 BIPMed raw dataset that supports this study's findings is available in EVA

6 repository/PRJEB39251, https://www.ebi.ac.uk/eva/?eva-study=PRJEB39251. ABraOM

7 raw dataset that supports the results of this study is available from ABraOM

8 (http://abraom.ib.usp.br/).

10 Acknowledgments

11 This work was supported by the Fundação de Amparo à Pesquisa do Estado de São

12 Paulo (FAPESP, grant number 2013/07559-3). RS was supported by FAPESP (grant

13 number 2019/08526-8). TKA is supported by FAPESP (grant number 2017/01900-6). IL-C

14 is supported by CNPq (grant number 311923/2019-4).

15

16 Competing interests

17 The authors declare no competing interests.

18

19 Author contributions

20 RS contributed with the study design, conceptualization, data acquisition, analysis, and

21 paper writing; TKA contributed with HLA sequencing, analysis, in silico prediction

22 analysis, and writing of the paper; MCG contributed with in silico prediction analysis and

23 paper writing; CSR contributed with public data acquisition and processing; $\mathrm{MN}$ and $\mathrm{MZ}$ 
1 contribute with public data acquisition; LD and MACB contributed with Belo Horizonte

2 data acquisition and sample information; VLV contributed with Barretos data acquisition

3 and sample information; WAS contributes with Ribeirão Preto data acquisition and sample

4 information; ILC contributed with project conceptualization and served as principal

5 investigators. All authors reviewed the manuscript. 


\section{References}

21 Shang, J. et al. Cell entry mechanisms of SARS-CoV-2. Proceedings of the

3 National Academy of Sciences 117, 11727-11734, doi:10.1073/pnas.2003138117 (2020).

52 Hou, Y. et al. New insights into genetic susceptibility of COVID-19: an ACE2 and TMPRSS2 polymorphism analysis. BMC Medicine 18, 216, doi:10.1186/s12916020-01673-z (2020).

$83 \mathrm{Lu}$, R. et al. Genomic characterisation and epidemiology of 2019 novel coronavirus:

9 implications for virus origins and receptor binding. The Lancet 395, 565-574,

10 doi:10.1016/S0140-6736(20)30251-8 (2020).

114 Simmons, G. et al. Inhibitors of cathepsin L prevent severe acute respiratory

12 syndrome coronavirus entry. Proceedings of the National Academy of Sciences of

13 the United States of America 102, 11876-11881, doi:10.1073/pnas.0505577102

$14 \quad$ (2005).

155 Hoffmann, M., Kleine-Weber, H. \& Pöhlmann, S. A Multibasic cleavage site in the 16 spike protein of SARS-CoV-2 is essential for infection of human lung cells.

$17 \quad$ Molecular Cell 78, 779-784.e775, doi:10.1016/j.molcel.2020.04.022 (2020).

186 Ye, Q., Wang, B. \& Mao, J. The pathogenesis and treatment of the 'Cytokine Storm'

19 in COVID-19. Journal of Infection 80, 607-613,

20 doi:https://doi.org/10.1016/j.jinf.2020.03.037 (2020).

217 Herold, T. et al. Elevated levels of IL-6 and CRP predict the need for mechanical 22 ventilation in COVID-19. Journal of Allergy and Clinical Immunology 146, 12823 136.e124, doi:10.1016/j.jaci.2020.05.008 (2020). 
18 Aziz, M., Fatima, R. \& Assaly, R. Elevated interleukin-6 and severe COVID-19: A

2 meta-analysis. Journal of Medical Virology n/a, doi:10.1002/jmv.25948 (2020).

39 Zhang, Q. et al. Inborn errors of type I IFN immunity in patients with life-

4 threatening COVID-19. Science, doi:10.1126/science.abd4570 (2020).

$5 \quad 10 \quad$ Cao, Y. et al. Comparative genetic analysis of the novel coronavirus (2019-

$6 \mathrm{nCoV} / \mathrm{SARS}-\mathrm{CoV}-2)$ receptor ACE2 in different populations. Cell Discov 6, 11,

7 doi:10.1038/s41421-020-0147-1 (2020).

811 Ellinghaus, D. et al. Genome-wide association study of severe covid-19 with

9 respiratory failure. New England Journal of Medicine,

10 doi:10.1056/NEJMoa2020283 (2020).

11 Zeberg, H. \& Pääbo, S. The major genetic risk factor for severe COVID-19 is

12 inherited from Neanderthals. Nature, doi:10.1038/s41586-020-2818-3 (2020).

1313 Shi, Y. et al. COVID-19 infection: the perspectives on immune responses. Cell

14 Death Differ 27, 1451-1454, doi:10.1038/s41418-020-0530-3 (2020).

1514 Nguyen, A. et al. Human leukocyte antigen susceptibility map for severe acute

16 respiratory syndrome coronavirus 2. Journal of Virology 94, e00510-00520,

17 doi:10.1128/JVI.00510-20 (2020).

1815 Rodrigues de Moura, R., Coelho, A. V. C., de Queiroz Balbino, V., Crovella, S. \&

19 Brandão, L. A. C. Meta-analysis of Brazilian genetic admixture and comparison

20 with other Latin America countries. American Journal of Human Biology 27, 674-

$21 \quad$ 680, doi:10.1002/ajhb.22714 (2015).

2216 Kehdy, F. S. G. et al. Origin and dynamics of admixture in Brazilians and its effect

23 on the pattern of deleterious mutations. Proceedings of the National Academy of

$24 \quad$ Sciences 112, 8696-8701, doi:10.1073/pnas.1504447112(2015). 
117 Lima-Costa, M. F. et al. Genomic ancestry and ethnoracial self-classification based

2 on 5,871 community-dwelling Brazilians (The Epigen Initiative). Sci Rep 5, 9812,

3 doi:10.1038/srep09812 (2015).

418 Secolin, R. et al. Distribution of local ancestry and evidence of adaptation in admixed populations. Sci Rep 9, 13900, doi:10.1038/s41598-019-50362-2 (2019).

619 Rocha, C. S., Secolin, R., Rodrigues, M. R., Carvalho, B. S. \& Lopes-Cendes, I. The Brazilian Initiative on Precision Medicine (BIPMed): fostering genomic datasharing of underrepresented populations. npj Genomic Medicine 5, 42, doi:10.1038/s41525-020-00149-6 (2020).

1020 Chapman, S. J. \& Hill, A. V. Human genetic susceptibility to infectious disease. Nat $11 \quad$ Rev Genet 13, 175-188, doi:10.1038/nrg3114 (2012).

1221 Karczewski, K. J. et al. The mutational constraint spectrum quantified from 13 variation in 141,456 humans. Nature 581, 434-443, doi:10.1038/s41586-020-2308-7 $14 \quad(2020)$.

1522 Auton, A. et al. A global reference for human genetic variation. Nature 526, 68-74, 16 doi:10.1038/nature15393 (2015).

1723 Novelli, A. et al. HLA alleles frequencies and susceptibility to COVID-19 in a 18 group of 99 Italian patients. HLA $\mathbf{n} / \mathbf{a}$, doi:10.1111/tan.14047 (2020).

1924 Naslavsky, M. S. et al. Exomic variants of an elderly cohort of Brazilians in the ABraOM database. Hum Mutat 38, 751-763, doi:10.1002/humu.23220 (2017).

2125 Wang, W., Zhang, W., Zhang, J., He, J. \& Zhu, F. Distribution of HLA allele frequencies in 82 Chinese individuals with coronavirus disease-2019 (COVID-19). HLA 96, 194-196, doi:10.1111/tan.13941 (2020). 
126 Dendrou, C. A., Petersen, J., Rossjohn, J. \& Fugger, L. HLA variation and disease.

$2 \quad$ Nat Rev Immunol 18, 325-339, doi:10.1038/nri.2017.143 (2018).

327 Alcendor, D. J. Racial disparities-associated COVID-19 mortality among minority

4 populations in the US. J Clin Med 9, doi:10.3390/jcm9082442 (2020).

528 Kaufman, H. W., Niles, J. K. \& Nash, D. B. Disparities in SARS-CoV-2 positivity

6 rates: Associations with race and ethnicity. Popul Health Manag,

$7 \quad$ doi:10.1089/pop.2020.0163 (2020).

829 Poulson, M. et al. National disparities in COVID-19 outcomes between black and

9 white Americans. J Natl Med Assoc, doi:10.1016/j.jnma.2020.07.009 (2020).

1030 Deng, L., Ruiz-linares, A., Xu, S. \& Wang, S. Ancestry variation and footprints of

11 natural selection along the genome in Latin American populations. Scientific

12 Reports 6, 1-7, doi:10.1038/srep21766 (2016).

1331 Cáceres, A. \& González, J. R. Following the footprints of polymorphic inversions

$14 \quad$ on SNP data: from detection to association tests. Nucleic acids research 43, e53-

15 e53, doi:10.1093/nar/gkv073 (2015).

1632 Homburger, J. R. et al. Genomic insights into the ancestry and demographic history

17 of South America. PLoS Genetics 11, doi:10.1371/journal.pgen.1005602 (2015).

1833 Danecek, P. et al. The variant call format and VCF tools. Bioinformatics 27, 2156-

19 2158, doi:10.1093/bioinformatics/btr330 (2011).

2034 Yates, A. D. et al. Ensembl 2020. Nucleic Acids Research 48, D682-D688,

21 doi:10.1093/nar/gkz966 (2020).

2235 Wang, K., Li, M. \& Hakonarson, H. ANNOVAR: functional annotation of genetic 23 variants from high-throughput sequencing data. Nucleic acids research 38, e164e164, doi:10.1093/nar/gkq603 (2010). 
136 McLaren, W. et al. The Ensembl variant effect predictor. Genome Biol 17, 122,

2 doi:10.1186/s13059-016-0974-4 (2016).

337 Mi, H., Muruganujan, A. \& Thomas, P. D. PANTHER in 2013: modelling the

4 evolution of gene function, and other gene attributes, in the context of phylogenetic

$5 \quad$ trees. Nucleic Acids Res 41, D377-386, doi:10.1093/nar/gks1118 (2013).

638 Schwarz, J. M., Rödelsperger, C., Schuelke, M. \& Seelow, D. MutationTaster

7 evaluates disease-causing potential of sequence alterations. Nat Methods 7, 575-

$8 \quad 576$, doi:10.1038/nmeth0810-575 (2010).

939 González-Pérez, A. \& López-Bigas, N. Improving the assessment of the outcome of non-synonymous SNVs with a consensus deleteriousness score, Condel. Am J Hum

11 Genet 88, 440-449, doi:10.1016/j.ajhg.2011.03.004 (2011).

1240 Choi, Y., Sims, G. E., Murphy, S., Miller, J. R. \& Chan, A. P. Predicting the

13 functional effect of amino acid substitutions and indels. PLoS One 7, e46688,

14 doi:10.1371/journal.pone.0046688 (2012).

1541 Adzhubei, I., Jordan, D. M. \& Sunyaev, S. R. Predicting functional effect of human

16 missense mutations using PolyPhen-2. Curr Protoc Hum Genet Chapter 7,

17 Unit7.20, doi:10.1002/0471142905.hg0720s76 (2013).

1842 Sim, N. L. et al. SIFT web server: predicting effects of amino acid substitutions on

19 proteins. Nucleic Acids Res 40, W452-457, doi:10.1093/nar/gks539 (2012).

2043 Tavtigian, S. V. et al. Comprehensive statistical study of 452 BRCA1 missense

21 substitutions with classification of eight recurrent substitutions as neutral. $J$ Med

22 Genet 43, 295-305, doi:10.1136/jmg.2005.033878 (2006). 
144 Rentzsch, P., Witten, D., Cooper, G. M., Shendure, J. \& Kircher, M. CADD:

2 predicting the deleteriousness of variants throughout the human genome. Nucleic

3 Acids Res 47, D886-D894, doi:10.1093/nar/gky1016 (2019).

445 Capriotti, E. \& Fariselli, P. PhD-SNPg: a webserver and lightweight tool for scoring single nucleotide variants. Nucleic Acids Res 45, W247-W252, doi:10.1093/nar/gkx369 (2017).

746 Shihab, H. A. et al. An integrative approach to predicting the functional effects of

1348 Rodrigues, C. H., Pires, D. E. \& Ascher, D. B. DynaMut: predicting the impact of 14 mutations on protein conformation, flexibility and stability. Nucleic Acids Res 46, 
152 Robinson, J. et al. IPD-IMGT/HLA database. Nucleic Acids Res 48, D948-D955, 2 doi:10.1093/nar/gkz950 (2020).

353 Excoffier, L. \& Lischer, H. E. Arlequin suite ver 3.5: a new series of programs to 4 perform population genetics analyses under Linux and Windows. Mol Ecol Resour $5 \quad$ 10, 564-567, doi:10.1111/j.1755-0998.2010.02847.x (2010).

654 Reynisson, B., Alvarez, B., Paul, S., Peters, B. \& Nielsen, M. NetMHCpan-4.1 and $7 \quad$ NetMHCIIpan-4.0: improved predictions of MHC antigen presentation by 8 concurrent motif deconvolution and integration of MS MHC eluted ligand data.

$9 \quad$ Nucleic Acids Res 48, W449-W454, doi:10.1093/nar/gkaa379 (2020).

1055 Nielsen, M., Justesen, S., Lund, O., Lundegaard, C. \& Buus, S. NetMHCIIpan-2.0 11 Improved pan-specific HLA-DR predictions using a novel concurrent alignment and 12 weight optimization training procedure. Immunome Res 6, 9, doi:10.1186/174513 7580-6-9 (2010).

1456 Gonzalez-Galarza, F. F. et al. Allele frequency net database (AFND) 2020 update: 15 gold-standard data classification, open access genotype data and new query tools. $16 \quad$ Nucleic Acids Res 48, D783-D788, doi:10.1093/nar/gkz1029 (2020). 


\section{Tables}

Table 1. Alternative allele frequency (AAF) of variants encompassing the COVID-19 risk core haplotype and the alleles present in Neanderthal samples.

\begin{tabular}{cccccccccccc}
\hline dbSNP & Chr & Pos & ALT & Brazil AF & Risk allele* & Campinas & Barretos & Ribeirão Preto & Belo Horizonte & BIPMed & ABraOM \\
\hline rs35044562 & 3 & 45909024 & G & 0.0011 & G & 0.0862 & 0.0000 & 0.0000 & 0.0000 & 0.0279 & 0.0311 \\
rs34326463 & 3 & 45899651 & G & 0.0011 & G & 0.0000 & 0.0000 & 0.0357 & 0.0000 & 0.0000 & 0.0000 \\
rs35508621 & 3 & 45880481 & C & 0.0011 & C & 0.0345 & 0.0000 & 0.0000 & 0.0000 & 0.0000 & 0.0039 \\
rs67959919 & 3 & 45871908 & A & 0.0011 & A & 0.0000 & 0.0000 & 0.0000 & 0.0000 & 0.0000 & 0.0039 \\
rs35624553 & 3 & 45867440 & G & 0.0262 & G & 0.0000 & 0.0000 & 0.0000 & 0.0000 & 0.0000 & 0.0039 \\
\hline
\end{tabular}

*data extracted from Zeberg and Pääbo, 2020 (doi:10.1038/s41586-020-2818-3) 
Table 2. Alternative allele frequency of deleterious variants according to 12 different prediction algorithms

\begin{tabular}{|c|c|c|c|c|c|c|c|c|c|c|c|c|}
\hline \multirow[b]{2}{*}{ Gene } & \multirow[b]{2}{*}{ Variant } & \multicolumn{11}{|c|}{ Alternative Allele Frequency } \\
\hline & & Campinas & Barretos & Ribeirão Preto & Belo Horizonte & $\mathrm{ABraOM}$ & BIPMed & NFE & AFR & AMR & EAS & SAS \\
\hline SLC6A20 & Phe249Ser & 0 & 0.0167 & 0 & 0 & 0.0008 & 0.0019 & 0.0005 & 0.0615 & 0.0003 & 0 & 0.0084 \\
\hline SLC6A20 & Gly164Val & 0 & 0 & 0 & 0 & 0.0008 & 0 & 0.0088 & 0 & 0 & 0 & 0 \\
\hline SLC6A20 & Leu25Pro & 0 & 0.0167 & 0 & 0 & 0 & 0 & 0 & 0 & 0 & 0 & 0 \\
\hline LZTFL1 & Leu96Arg & 0 & 0 & 0 & 0 & 0.0008 & 0 & 0 & 0 & 0 & 0 & 0 \\
\hline$X C R 1$ & Tyr287Ser & 0 & 0 & 0 & 0.0167 & 0 & 0 & 0 & 0 & 0 & 0 & 0 \\
\hline FURIN & Gly146Ser & 0 & 0 & 0 & 0 & 0.0008 & 0 & 0.0004 & 0.0002 & 0.0005 & 0.0544 & 0.0327 \\
\hline FURIN & Asn414Ser & 0 & 0 & 0 & 0 & 0 & 0.0019 & 0 & 0 & 0 & 0 & 0 \\
\hline
\end{tabular}

$\mathrm{NFE}=$ non-Finish European; AFR=sub-Saharan African/African American; AMR=admixed Americans/Latinos; EAS=east Asians; SAS=south Asians. 
Table 3. List of HLA strongest binders ( $>300$ peptides bound at high affinity) of SARS-CoV-2 peptides and frequency in the Brazilian sample.

\begin{tabular}{|c|c|c|}
\hline HLA & HLA alleles & Allele frequency \\
\hline \multirow{20}{*}{ HLA -A } & $A^{*} 01: 01$ & 0.10233 \\
\hline & A*11:01 & 0.04145 \\
\hline & $A * 11: 67$ & 0.00130 \\
\hline & $A * 23: 01$ & 0.03756 \\
\hline & $A * 23: 17$ & 0.00389 \\
\hline & $A * 24: 02$ & 0.10104 \\
\hline & $A * 24: 03$ & 0.00259 \\
\hline & $A * 24: 05$ & 0.00130 \\
\hline & $A * 25: 01$ & 0.00389 \\
\hline & $A * 26: 01$ & 0.02979 \\
\hline & $A * 26: 02$ & 0.00130 \\
\hline & $A * 26: 08$ & 0.00130 \\
\hline & $A * 29: 01$ & 0.00259 \\
\hline & $A * 29: 02$ & 0.04534 \\
\hline & $A * 29: 119$ & 0.00130 \\
\hline & $A * 30: 02$ & 0.02591 \\
\hline & $A * 30: 04$ & 0.00259 \\
\hline & $A * 34: 02$ & 0.00777 \\
\hline & $A * 36: 01$ & 0.00389 \\
\hline & $A * 80: 01$ & 0.00130 \\
\hline \multirow{2}{*}{ HLA-B } & $B * 15: 08$ & 0.00130 \\
\hline & $\mathrm{B} * 15: 11$ & 0.00130 \\
\hline \multirow{5}{*}{ HLA-C } & $C^{*} 03: 02$ & 0.00389 \\
\hline & $C * 07: 02$ & 0.05699 \\
\hline & $C^{*} 07: 50$ & 0.00130 \\
\hline & $C^{*} 14: 02$ & 0.02979 \\
\hline & $C^{*} 14: 03$ & 0.00259 \\
\hline \multirow{4}{*}{ HLA-DQ } & DQA1*0201-DQB1*0402 & 0.00259 \\
\hline & DQA1*0301-DQB1*0402 & 0.00130 \\
\hline & DQA1*0303-DQB1*0401 & 0.00130 \\
\hline & DQA $1 * 0303-\mathrm{DQB} 1 * 0402$ & 0.00130 \\
\hline
\end{tabular}




\section{Figures legend}

Figure 1. Distribution of alternative allele frequency of common variants (AAF $\geq 0.01)$ from samples of admixed Brazilians and worldwide public datasets. The $\mathrm{x}$-axis shows the 56 variants found in common between the Brazil sample and the gnomAD and $1 \mathrm{KGP}$ dataset. (A) Comparison between Brazilians and gnomAD, and (B) including non-Finland Europeans (NFE), sub-Saharan Africans/African Americans (AFR) Venn diagrams that show the overlap between samples (A) and variants (B) in the WES and the SNP array datasets from BIPMed reference samples.

Figure 2. Distribution of alternative allele frequency of common variants $(\mathrm{AAF}>0.01)$ separated by Brazilian cities. The $\mathrm{x}$-axis shows the 56 variants found in common with gnomAD and $1 \mathrm{KGP}$. This barplot also includes the two public Brazilian datasets (BIPMed and ABraOM) and the frequency of all samples combined (Brazil). 
bioRxiv preprint doi: https://doi.org/10.1101/2020.12.04.411736; this version posted December 6. 2020. The copyright holder for this preprint (which was not certified by peer review) is the author/funder, who has granted bioRxiv a license to display the preprint in perpetuity. It is made available under aCC-BY-NC-ND 4.0 International license.

Figure 1
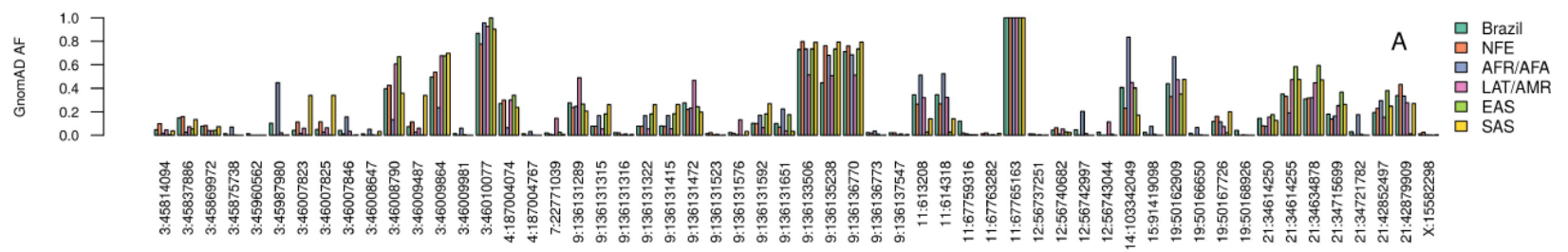

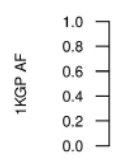

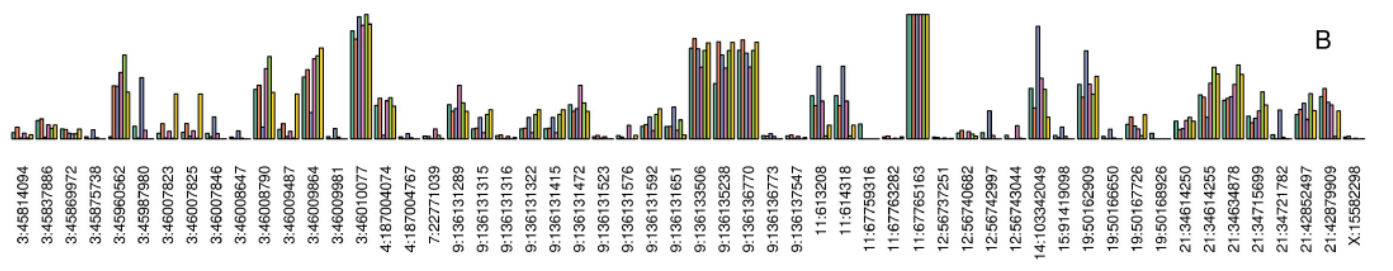

Figure 2
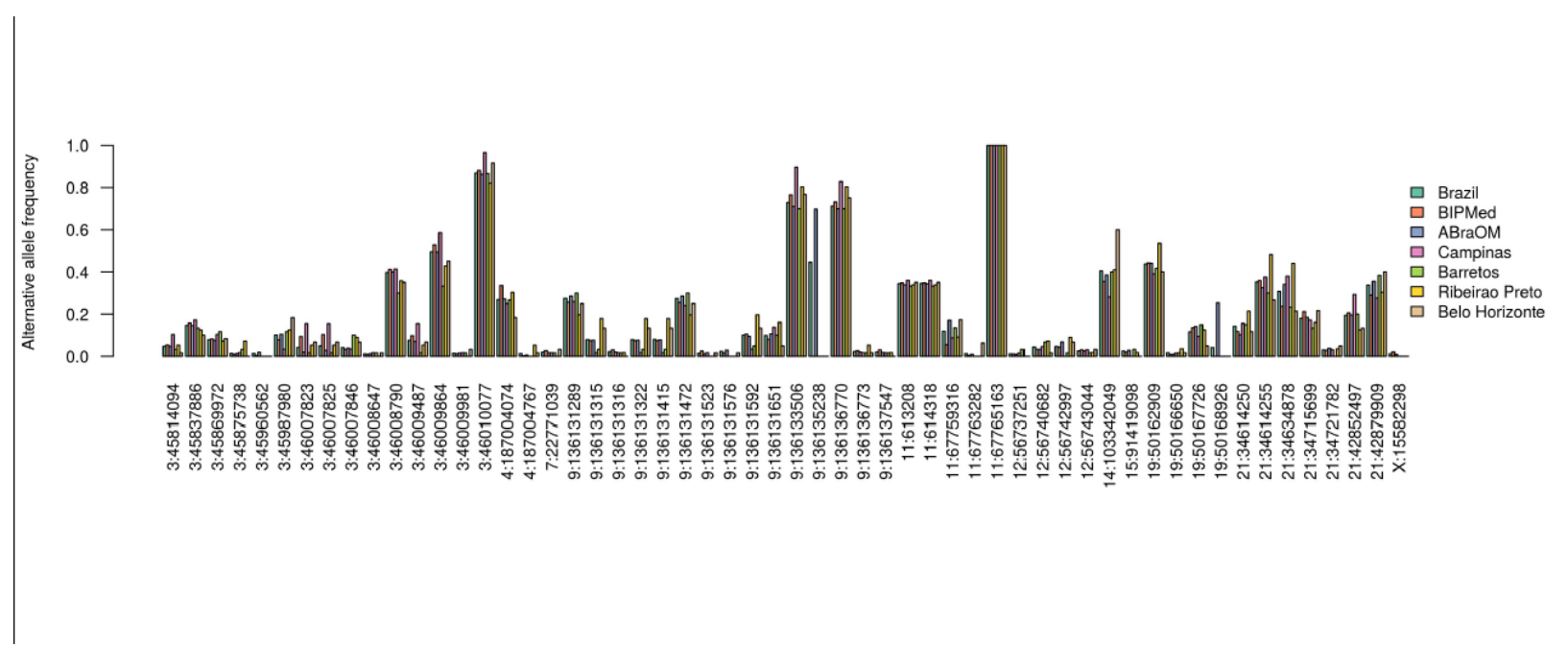